india/en/home/projects/ gavil.html

43. $67 \%$ surveyed Indians have developed antibodies against coronavirus, 40 crore still at risk: Govt [Internet]. India Today. [cited 2021 Dec 16]. Available from: https://www. indiatoday.in/ coronavirus-outbreak/story/fourth-serosurvey-result-67-per cent-indians-developed-antibodies-against-covid19-18304432021-07-20

44. Cavanaugh AM, Spicer KB, Thoroughman D, et al. Reduced risk of reinfection with SARS-CoV-2 after COVID-19 vaccination Kentucky, May-June 2021. Morb Mortal Wkly Rep. 2021;70:1081-3.

45. Bozio CH, Grannis SJ, Naleway AL, et al. Laboratory-confirmed COVID-19 among adults hospitalized with COVID-19-Like illness with infection-induced or mRNA vaccine-induced SARSCoV-2 immunity - nine states, January-September 2021. Morb Mortal Wkly Rep. 2021;70:1539-44.

\section{NEWS INBRIEF}

\section{Effect of mixing the vaccines against Covid-19}

Vaccines trigger the immune response by imitating an infection, leading to the generation of the memory T-cells and B-cells. This antibody response gets enhanced with the second dose and boosters leading to the generation of antibody levels which will be protective against future infections.But does this response appear / persist, if the type of vaccine has been changed for the second dose?

In a recently published paper by Com-COV-2 Study Group - a multicentre survey network of nine institutions in the UK, researchers studied the effect of mixing of different COVID-19 vaccines on the antibody response. In this study, 1072 participants were studied where the participants were inoculated with a dose of the Pfizer- BioNTech mRNA (BNT162b2), Moderna mRNA (mRNA-1273), Astra Zeneca (chimpanzee nonreplicating adenovirus (ChAdOx1 nCoV-19), or Novavax Matrix M-adjuvanted recombinant S protein (NVX-CoV2373) vaccine after an initial dose of Astra Zeneca or Pfizer. Higher levels of binding and neutralizing antibodies were seen with the second dose of Moderna vaccine after a first dose of Astra Zeneca or Pfizer compared to two doses of either Pfizer or Astra Zeneca.

Findings of the present study, provide the data to support the mix and match of COVID-19 vaccines in primary immunization schedules. This will provides the much needed flexibility required to vaccinate the large unvaccinated population in low income countries.

With the emergence of new variant Omicron, it is the need of hour to vaccinate as many people as possible (BMJ 07 December, 2021)

\section{Finally something against childhood dental caries}

Exact burden of dental caries in India is not known due to poor awareness among the general public about its long term impact on health affecting the growth, early childhood development, learning and limited published literature. A recent meta-analysis estimated that the approxi-mately $52 \%$ of children aged 3-18 years have caries in India.

In the absence of a validated risk determining tool, prevention and screening are the best modalities. Recently, United States Preventive Services Task Force (USPSTF) has recommended that the primary care physicians must prescribe oral fluoride supplements to all asymptomatic children aged 6 months to 5 years, living in areas having lesser than $0.6 \mathrm{ppm}$ fluoride levels (fluoride deficient areas). Use of fluoride varnish containing 5\% sodium fluoride is also recommended to all childrenaged 6 months to 5 years after the eruption of primary teeth. These recommendations are beyond the routine dental evaluation and referral to the dental health professionals.

More Indian data is needed before recommendations in Indian context are produced to reduce the potentially preventable burden of dental caries in Indian children.

(JAMA 07 December, 2021)

\section{Microfluidics: Future of treatment of Neonatal Jaundice}

Neonatal jaundice is the most common morbidity in the first week of life after birth. Almost $60 \%$ of term and $80 \%$ of preterm babies develop jaundice. Approximately $5-8 \%$ of these babies require one or another modality to lower the serum bilirubin levels, in order to prevent neurological damage. Beyond particular levels or in the presence of features suggestive of bilirubin encephalopathy, double volume exchange transfusion (DVET) rapidly lowers the bilirubin levels but in VLBW/ELBW babies it can cause hemodynamic instability.

Researchers at Oregon State University College of Engineering has led to a promising potential therapy for the treatment of neonatal jaundice using microfluidics. Microfluidics is the branch of science which study the behavior of fluids, as they travel through or are confined in microminiaturized devices equipped with channels and chambers. The team has found a simpler and safer alternative to DVET, by treating the patient's blood by circulating it through an external device known as a microfluidic photoreactor. The basic principle is same as that of phototherapy but using the microfluidics helps in lowering the bilirubin at faster rates. Preclinical studies in Gunn rats, using high-intensity light at $470 \mathrm{~nm}$ for 4 hours demonstrates a significant reduction in the bilirubin levels without causing an appreciable DNA damage. The rates of bilirubin reduction were similar to those observed with exchange transfusion and on a similar time scale. Mathematical prediction model for the human newborn, suggested that this newer modality will outperform the exchange transfusion at the clinical scale.

Use of microfluidics is a potential promising approach for the treatment of neonatal jaundice, especially in the VLBW/ ELBW, babies without the use of donor blood.

(Biomicrofluidics 24 November, 2021)

Rajesh Kumar Meena raj.mamc@gmail.com 